\title{
HIFU for the treatment of gastric cancer with liver metastases with unsuitable indications for hepatectomy and radiofrequency ablation: a prospective and propensity score-matched study Running title: HIFU treatment for gastric cancer with liver metastases
}

\section{Lihu Gu}

HwaMei Hospital, University of Chinese Academy of Sciences

\section{Ning He}

HwaMei Hospital, University of Chinese Academy of Sciences

\section{Jiaze Hong}

Zhejiang Chinese Medical University

\section{Tong Yang}

HwaMei Hospital, University of Chinese Academy of Sciences

\section{Derry $\mathbf{N g}$}

Medical College of Ningbo University

\section{Xudong Gao}

HwaMei Hospital, University of Chinese Academy of Sciences

Kun Yan

HwaMei Hospital, University of Chinese Academy of Sciences

\section{Xiaoxiang Fan}

HwaMei Hospital, University of Chinese Academy of Sciences

\section{Zhi Zheng}

HwaMei Hospital, University of Chinese Academy of Sciences

\section{Ping Chen}

HwaMei Hospital, University of Chinese Academy of Sciences

Jianjun Zheng

HwaMei Hospital, University of Chinese Academy of Sciences

Qi Zheng ( $\square$ hifuno2hospital@163.com )

HwaMei Hospital, University of Chinese Academy of Sciences

\section{Research Article}

Keywords: high intensity focused ultrasound, gastric cancer with liver metastases, palliative chemotherapy, best supportive care, prognosis

Posted Date: April 14th, 2021

DOI: https://doi.org/10.21203/rs.3.rs-401693/v1 
License: @ (i) This work is licensed under a Creative Commons Attribution 4.0 International License. Read Full License

Version of Record: A version of this preprint was published at BMC Surgery on July 12th, 2021. See the published version at https://doi.org/10.1186/s12893-021-01307-y. 


\section{Abstract}

Background: The purpose of this study was to explore the efficacy and safety of high intensity focused ultrasound (HIFU) in gastric cancer with liver metastasis (GCLM) patients who were contraindicated for either hepatectomy or radiofrequency ablation (RFA).

Methods: This is a prospective, observational study on GCLM patients with 1-3 liver metastases. The primary gastric lesions were thoroughly resected and any case that exhibited extra-hepatic metastasis was excluded. A 1:2:2 propensity score-matching analysis was performed using a logistic regression model on the HIFU group, best supportive care (BSC) group, and palliative chemotherapy (PC) group. The primary endpoints include progression-free survival (PFS) and overall survival (OS).

Results: Forty patients were finally included, there were 8 cases in HIFU group, 16 cases in BSC group, and 16 cases in PC group. The median follow-up time for the entire cohort was 10 months. The median PFS was 16.5 months in HIFU group, 2 months in BSC group, and 5 months in PC group. The median OS was 27.5 months in the HIFU group, 7 months in the BSC group, and 11.5 months in the PC group. Additionally, no grade 3 or higher adverse events occurred in the HIFU group.

Conclusion: The results of this study showed that HIFU treatment could improve the long-term prognosis of GCLM patients without a significant increase in the occurrence of adverse events. Compared with PC and BSC, HIFU is the preferred treatment option when GCLM patients without extra-hepatic metastasis are unable to undergo either surgery or RFA.

\section{Introduction}

Gastric cancer (GC) is the fifth most commonly diagnosed malignancy and the third leading cause of death from cancer worldwide[1]. The main reason for the high mortality rate is that the prognosis of advanced GC is usually poor, especially in patients with distant metastasis[2]. The liver is one of the most common metastatic sites from GC. About $10 \%$ of patients with GC are initially diagnosed with liver metastasis and liver metastases occur in about $37 \%$ of patients after radical gastrectomy[3,4]. Previous studies have shown that systemic chemotherapy is the recommended treatment for patients with gastric cancer with liver metastasis (GCLM)[5]. However, there is growing evidence that when the metastatic lesions are limited to the liver, local treatments such as hepatectomy and radiofrequency ablation (RFA) are viable alternative therapies[6, 7]. It has been reported that the 5-year overall survival (OS) of patients with GCLM without extra-hepatic metastasis is increased by $10-30 \%$ after successful surgical resection of the liver metastasis $[8,9]$. Similarly, RFA can achieve a better long-term effect in selected patients with GCLM, with a 5 -year OS of 3\%-30\%[10, 11].

Unfortunately, due to various reasons, a considerable number of patients with GCLM cannot receive surgery or RFA. Generally speaking, this group of patients can only receive palliative chemotherapy (PC) or best supportive care (BSC), and either choice has a poor prognosis. The median survival time of these patients is typically less than a year[12, 13].

In recent years, a new local treatment technique, high-intensity focused ultrasound (HIFU), has been used for the treatment of liver tumors, which includes both primary and metastatic liver cancers. During HIFU treatment, ultrasound waves are focused and hyperthermia up to $60-100^{\circ} \mathrm{C}$ is induced in the targeted lesion. Within this temperature range, the water within the tissues begin to vaporize and microbubble formation happens after. Cavitation then ensures and cell death occurs through the process of coagulative necrosis[14, 15]. Previous studies have shown that HIFU treatment could treat, including but not limited to liver tumors and pancreatic cancer, and is associated with fewer serious adverse reactions $[16,17]$. According to our previous studies, HIFU treatment for primary liver cancer and colorectal liver metastases has achieved surprising positive results[18, 19]. Therefore, we designed a prospective, propensity score 
matching study for patients with GCLM without evidence of extrahepatic metastases who were unable to undergo hepatectomy or RFA.

\section{Methods And Materials}

\section{Study design}

This was a single-institution, prospective observational study to evaluate the efficacy and safety of HIFU treatment compared with BSC and PC for patients with GCLM. The database comprises data collected from GC patients from January 2014 to December 2019 at HwaMei Hospital, University of Chinese Academy of Sciences. This study was approved by the research ethics committee of HwaMei Hospital, University of Chinese Academy of Sciences (approval NO. PJ-NBEY-KY-2019-153-01). Written consent was obtained from all patients before enrollment. Patients were eligible for the study if they had liver metastases (synchronous liver metastasis, metachronous liver metastasis) from GC, and their primary lesions (gastric lesions) had been removed without evidence of extrahepatic metastasis. Patients were evaluated by HwaMei Hospital, University of Chinese Academy of Sciences multiple disciplinary team (MDT), which comprises gastrointestinal surgeons, hepatobiliary surgeons, medical oncologists, interventional radiologists, hepatologists, and HIFU oncologists. Unsuitable indications of resection include the following: determined unresectable by MDT, resectable but is considered a major or difficult surgery for patients who are in poor condition and patients who refused surgery. Metastases were judged as unsuitable for RFA due to their proximity to vessels, bile ducts, the gastrointestinal tract or gallbladder, the diaphragm, or large size $(>3 \mathrm{~cm})$, etc.

Exclusion criteria: 1) The patient refused to participate in the study. 2) The pathology of gastric lesions was not gastric adenocarcinoma or the liver lesions were the primary liver cancer. 3) The number of liver metastases was more than three. 4) The patient was lost to follow-up, or the data were incomplete. Other key eligibility criteria include an Eastern Cooperative Oncology Group (ECOG) performance score of $\leq 2$, adequate organ and bone marrow function, defined as having a white blood cell count $>3.5 \times 10^{3} / \mathrm{uL}$, neutrophil count $>1500 / \mathrm{uL}$, hemoglobin $>10 \mathrm{~g} / \mathrm{dL}$, creatinine $\leq 2 \times$ upper limit of normal (ULN), creatinine clearance $60 \mathrm{~mL} / \mathrm{min} / 1.73 \mathrm{~m}$, alanine aminotransferase (ALT) or aspartate aminotransferase (AST) $<2 \times U L N$, bilirubin $\leq 1.5 \times U L N$, and platelets $\geq 100 \times 10^{9} / \mathrm{L}$. The additional exclusion criteria for this study are as follows: congestive heart failure, unstable angina, active cardiomyopathy, unstable ventricular arrhythmia, previous stroke $<12$ months, human immunodeficiency virus, and other active malignancies. The primary endpoints include progression-free survival (PFS) and OS.

Patients were classified according to the treatment received for their liver metastases. The first group was treated with HIFU (HIFU group), regardless of prior treatment with or without other local treatments (such as chemotherapy, targeted therapy, RFA, transcatheter arterial chemoembolization (TACE), immunotherapy, etc.) for liver metastases, or continued with other treatments after the completion of HIFU treatment. The second group received BSC for liver metastases without receiving any other anti-tumor treatments (BSC group), but there was no restriction on receiving adjuvant chemotherapy after radical gastrectomy was performed. The third group only received PC for liver metastases (PC group) and did not receive additional relevant anti-tumor therapy (such as targeted therapy, RFA, TACE, immunotherapy, etc.). The chemotherapy regimen used is based on 5-fluorouracil (5-FU) or platinum.

\section{HIFU Treatment}

The JC200 + HIFU system (Chongqing Haifu Technology, Chongqing, China) was used for this research. In summary, it has a transducer with a diameter of $20 \mathrm{~cm}$, a focal length of $16.5 \mathrm{~cm}$, and an operating frequency of $1.0 \mathrm{MHz}$ with a focal region of $3 \times 3 \times 8 \mathrm{~mm}$. Refer to the previous study for operational details[18]. A treatment plan was devised with a 3dimensional reconstruction of the tumor boundary with 5-mm-separated sections. Typically, the planned treatment volume should exceed the tumors' margin by at least $5 \mathrm{~mm}$. 
Systemic therapy can be continued one week after the operation according to the needs of the disease. The adjuvant therapy regimen for all patients was determined by the oncologist or MDT.

\section{Follow-up}

Adverse events (AEs) were graded using the National Cancer Institute Common Terminology Criteria for Adverse Events (CTCAE), version 4.0[20]. In the HIFU group, the tumor responses were assessed using the modified Response Evaluation Criteria in Solid Tumors (mRECIST)[21]. Complete response (CR): the disappearance of any intratumoral arterial enhancement in all target lesions. Partial response (PR): at least a 30\% decrease in the sum of diameters of viable (contrast enhancement in the arterial phase) target lesions, taking as reference the baseline sum of the diameters of target lesions. Progressive disease (PD): an increase of at least $20 \%$ in the sum of the diameters of viable (enhancing) target lesions, taking as reference the smallest sum of the diameters of viable (enhancing) target lesions that were recorded just prior to the initiation of the treatment. Stable disease (SD): any cases that do not qualify for either PR or PD. Tumor response was assessed on contrast-enhanced MRI or CT and was carried out on day 30 and every 3 months thereafter.

In both the BSC and PC groups, tumor response was determined according to the Response Evaluation Criteria in Solid Tumors (RECIST)[22]. PFS is defined as the time from the first HIFU treatment/BSC/PC to death, locoregional recurrence, or distant recurrence. OS is defined as the time from completion to the first HIFU/BSC/PC to death from any cause or lost follow-up. The median follow-up time for the entire cohort was 10 months (range 3-44 months), and follow-up of all patients included in this study was stopped on December 2020.

\section{Statistical analysis}

To reduce selection bias, a 1:2:2 propensity score matching analysis was performed on the HIFU group, BSC group, and PC group. Propensity scores were estimated using a logistic regression model and the following covariates: age, gender, body mass index (BMI), ECOG, Child-Pugh class, the time of liver metastases, the number and size of liver lesions, TNM stage of GC, the number of lymph nodes retrieved. Using these propensity scores, patients with HIFU treatment (HIFU group) were individually matched to patients with BSC (BSC group) and patients with PC (PC group)[23].

Hazard ratios (HR) and 95\% confidence intervals (Cl) were calculated. Time to progression and survival was evaluated using the Kaplan-Meier method. PFS was calculated using the Kaplan-Meier method, and the log-rank test was employed to determine the significance. Continuous variables were compared using the independent-samples t-test or Wilcoxon rank-sum test, and categorical variables were compared using Pearson's chi-squared test or Fisher's exact test, when appropriate. All statistical tests were performed 2-sided, and $p<0.05$ was considered statistically significant. Analyses were performed using SPSS software (version 25.0, SPSS Inc. IL, USA).

\section{Results}

A total of $960 \mathrm{GC}$ patients underwent gastrectomy at HwaMei Hospital, University of Chinese Academy of Sciences between January 2014 and December 2019, of which 331 patients were diagnosed with liver metastasis from GC during treatment, and were follow-up with during this study. After the selection process was performed based on the abovementioned inclusion and exclusion criteria, a total of 172 GCLM patients initially met the conditions of this study, there were 8 patients in the HIFU group, 55 patients in the BSC group, and 109 patients in the PC group. After performing a 1:2:2 propensity matching analysis, 40 patients were finally selected, there were 8 patients in the HIFU group, 16 patients in the BSC group, and 16 patients in the PC group. The entire flowchart is described in Fig. 1. All 40 patients (70 lesions) were unable to receive surgery or RFA, please refer to Supplementary Table 1 for the specific reasons. Among the three 
groups, it was found that there was no statistically significant difference in most of the covariates as detailed in Table 1-2. 
Table 1

Basic information of the patients.

\begin{tabular}{|c|c|c|c|c|}
\hline Characteristic & HIFU $(N=8)$ & $\operatorname{BSC}(N=16)$ & $P C(N=16)$ & $\mathrm{p}$ value \\
\hline Age (years) (mean $\pm S D)$ & $63.9 \pm 5.6$ & $64.1 \pm 5.1$ & $62.8 \pm 4.9$ & $>0.1$ \\
\hline Gender & & & & $>0.1$ \\
\hline Male & 6 & 8 & 6 & \\
\hline Female & 2 & 8 & 10 & \\
\hline $\operatorname{BMI}\left(\mathrm{kg} / \mathrm{m}^{2}\right)(\operatorname{mean} \pm \mathrm{SD})$ & $19.8 \pm 2.4$ & $18.3 \pm 2.1$ & $20.5 \pm 1.5$ & $>0.1$ \\
\hline Comorbidity & & & & $>0.1$ \\
\hline No & 2 & 7 & 8 & \\
\hline Yes & 6 & 9 & 8 & \\
\hline ECOG performance status & & & & $>0.1$ \\
\hline 0 & 4 & 4 & 6 & \\
\hline 1 & 4 & 10 & 9 & \\
\hline 2 & 0 & 2 & 1 & \\
\hline Child-Pugh class & & & & $>0.1$ \\
\hline Grade A & 7 & 13 & 11 & \\
\hline Grade B & 1 & 3 & 5 & \\
\hline Liver metastases & & & & $>0.1$ \\
\hline Synchronous & 2 & 1 & 1 & \\
\hline Metachronous & 6 & 15 & 15 & \\
\hline First-line treatment of liver metastases & & & & NA \\
\hline Chemotherapy & 2 & - & 16 & \\
\hline RFA & 2 & - & 0 & \\
\hline HIFU + TACE & 2 & - & 0 & \\
\hline HIFU & 2 & - & 0 & \\
\hline Chemotherapy before surgery & & & & 0.09 \\
\hline No & 6 & 16 & 15 & \\
\hline Yes & 2 & 0 & 1 & \\
\hline TNM stage of GC & & & & $>0.1$ \\
\hline I & 1 & 4 & 2 & \\
\hline II & 1 & 3 & 4 & \\
\hline
\end{tabular}

HIFU, high intensity focused ultrasound; BSC, best supportive care; PC, palliative chemotherapy; BMI, body mass index; ECOG, Eastern Cooperative Oncology Group; RFA, radiofrequency ablation; TACE, transcatheter arterial chemoembolization; GC, gastric cancer; NA, not available. 


\begin{tabular}{|c|c|c|c|c|}
\hline Characteristic & HIFU $(\mathrm{N}=8)$ & $\operatorname{BSC}(\mathrm{N}=16)$ & $P C(N=16)$ & p value \\
\hline IIII & 4 & 8 & 9 & \\
\hline IV & 2 & 1 & 1 & \\
\hline Number of lymph nodes retrieved, Median (IQR) & $19(12-26)$ & $18(12-27)$ & $20(13-25)$ & $>0.1$ \\
\hline
\end{tabular}


Table 2

Liver metastases' characteristic.

\begin{tabular}{|c|c|c|c|c|}
\hline Characteristic & HIFU $(N=8)$ & $\operatorname{BSC}(\mathrm{N}=16)$ & $P C(N=16)$ & $p$ value \\
\hline Previous lines of liver metastases therapy & & & & NA \\
\hline Nil & 4 & 16 & 16 & \\
\hline 1 & 3 & 0 & 0 & \\
\hline 2 & 1 & 0 & 0 & \\
\hline $\begin{array}{l}\text { Time from initial diagnosis to HIFU (month), } \\
\text { (range) }\end{array}$ & $0-7$ & - & - & \\
\hline Number & & & & $>0.1$ \\
\hline 1 & 5 & 8 & 8 & \\
\hline 2 & 1 & 4 & 3 & \\
\hline 3 & 2 & 4 & 5 & \\
\hline Maximum size (cm), Median (range) & $2.0(1.2-8.8)$ & $\begin{array}{l}3.8(1.0- \\
8.0)\end{array}$ & $3.0(1.0-7.0)$ & $>0.1$ \\
\hline \multicolumn{5}{|l|}{ Location (liver segment) } \\
\hline I & 0 & 2 & 1 & \\
\hline$\|$ & 1 & 1 & 3 & \\
\hline III & 1 & 1 & 2 & \\
\hline IV & 1 & 5 & 1 & \\
\hline V & 1 & 3 & 4 & \\
\hline VI & 4 & 7 & 6 & \\
\hline VII & 1 & 1 & 3 & \\
\hline VIII & 2 & 2 & 4 & \\
\hline \multicolumn{5}{|l|}{ Systematic treatment after HIFU } \\
\hline No & 1 & - & - & \\
\hline Yes & 7 & - & - & \\
\hline Input energy of HIFU (kJ), Median(IQR) & $625(353-848)$ & - & - & \\
\hline Input power of HIFU (W), Median(IQR) & $340(297-360)$ & - & - & \\
\hline Cumulative transmit time of HIFU (s), Median(IQR) & $\begin{array}{l}1890(1230- \\
2219)\end{array}$ & - & - & \\
\hline
\end{tabular}

Among the 40 patients, there was an equal proportion of males and females. The vast majority (92.5\%) of patients had an ECOG of $0-1$, and only three had an ECOG of 2. All participants had a liver function of Child-Pugh A or B, with $77.5 \%$ of patients having a liver function of Child-Pugh A. Liver metastases were present at the initial primary diagnosis in $10 \%$ 
of patients (four cases). Although there was no statically significant difference among the three groups, the rate of synchronous liver metastasis in the HIFU group was higher than that of the other two groups $(25 \%, 6.25 \%, 6.25 \%$, respectively). Three patients ( 2 in the HIFU group and 1 in the PC group) received chemotherapy before surgery. The postsurgery pathological staging of GC showed that $17.5 \%$ of patients were in stage I, $72.5 \%$ were in stage II-III, and $10 \%$ were in stage IV, the latter presented with liver metastasis only. Details are described in Table 1.

In the HIFU group, half of the patients received line 1 or above treatment. The other two groups of patients did not receive any additional anti-tumor therapy due to liver metastasis. Approximately half of the patients had a single lesion, eight had two lesions and 11 had three lesions. Most patients (87.5\%) received systematic treatment after HIFU was performed. More details are described in Table 2. All patients successfully underwent HIFU treatment without any complications during the operation.

\section{Long-term Outcome}

The median PFS was 16.5 months in the HIFU group, 2 months in the BSC group, and 5 months in the PC group. KaplanMeier curves were used to determine the PFS of the HIFU group, BSC group, and PC group. The PFS of the HIFU group was significantly higher than that of the other two groups, while the PFS of the PC group was significantly higher than that of the BSC group (Fig. 2). The median OS was 27.5 months in the HIFU group, 7 months in the BSC group, and 11.5 months in the PC group. Among the three groups, the patients who received HIFU had the best OS, followed by the patients who received PC, and the patients who received BSC had the worst OS (Fig. 3). At the end of the follow-up period, 11 patients $(27.5 \%)$ were still alive, of whom 3 had no evidence of recurrence (2 in the HIFU group, 1 in the PC group) and 29 died(72.5\%) from tumor progression. No patients were lost to follow-up.

\section{Tumor Response}

Evaluation of therapeutic response with contrast-enhanced MRI was performed 30 days after the HIFU treatment. All lesions (a total of 13 ) were suitable for assessment. Five patients (62.5\%) achieved CR and two achieved PR, the remaining patient achieved SD. The objective response rate (ORR) was $87.5 \%$. At the time of data analysis, two patients were alive at 26 and 29 months without any evidence of recurrence. Four developed liver recurrence or distant metastasis and were alive. Two patients with recurrence died due to tumor progression (Table 3). 
Table 3

Patient's details.

\begin{tabular}{|c|c|c|c|c|c|c|c|c|c|}
\hline No. & Gender & Age & ECOG & $\begin{array}{l}\text { Previous } \\
\text { lines of } \\
\text { liver } \\
\text { metastases } \\
\text { therapy }\end{array}$ & $\begin{array}{l}\text { HIFU } \\
\text { treatment }\end{array}$ & $\begin{array}{l}\text { Tumor } \\
\text { response\# }\end{array}$ & $\begin{array}{l}\text { Systematic } \\
\text { treatment } \\
\text { after HIFU }\end{array}$ & $\begin{array}{l}\text { Recurrence } \\
\text { after first } \\
\text { HIFU }\end{array}$ & $\begin{array}{l}\text { Outcome } \\
\text { (month) }\end{array}$ \\
\hline 1 & Male & 71 & 0 & - & HIFU & PR & Tegafur & Hepatic & $\begin{array}{l}\text { Expired } \\
(24)\end{array}$ \\
\hline 2 & Female & 67 & 1 & - & HIFU & $\mathrm{CR}$ & Tegafur & Extrahepatic & $\begin{array}{l}\text { Alive with } \\
\text { recurrence } \\
\text { (44) }\end{array}$ \\
\hline 3 & Male & 62 & 0 & - & $\begin{array}{l}\text { TACE + } \\
\text { HIFU }\end{array}$ & $\mathrm{CR}$ & - & Hepatic & $\begin{array}{l}\text { Alive with } \\
\text { recurrence } \\
\text { (44) }\end{array}$ \\
\hline 4 & Female & 55 & 1 & RFA & HIFU & $\mathrm{CR}$ & $\begin{array}{l}\text { Paclitaxel } \\
+ \text { Tegafur }\end{array}$ & Extrahepatic & $\begin{array}{l}\text { Alive with } \\
\text { recurrence } \\
\text { (41) }\end{array}$ \\
\hline 5 & Male & 62 & 1 & RFA; TACE & HIFU & $\mathrm{CR}$ & Tegafur & - & $\begin{array}{l}\text { Alive } \\
\text { without } \\
\text { recurrence } \\
(29)\end{array}$ \\
\hline 6 & Male & 71 & 0 & sox & HIFU & CR & sox & - & $\begin{array}{l}\text { Alive } \\
\text { without } \\
\text { recurrence } \\
(26)\end{array}$ \\
\hline 7 & Male & 64 & 1 & - & $\begin{array}{l}\text { TACE + } \\
\text { HIFU }\end{array}$ & SD & $\begin{array}{l}\text { Nab- } \\
\text { paclitaxel } \\
+ \text { Tegafur }\end{array}$ & Extrahepatic & $\begin{array}{l}\text { Expired } \\
(11)\end{array}$ \\
\hline 8 & Male & 59 & 0 & SOX & HIFU & PR & - & Hepatic & $\begin{array}{l}\text { Alive with } \\
\text { recurrence } \\
\text { (6) }\end{array}$ \\
\hline $\begin{array}{l}\text { ECO } \\
\text { TAC } \\
\text { perf }\end{array}$ & $\begin{array}{l}\text { Eastern } \\
\text { ranscath } \\
\text { ned at } 3\end{array}$ & $\begin{array}{l}\text { oper } \\
\text { ter ar } \\
\text { days }\end{array}$ & $\begin{array}{l}\text { ve Once } \\
\text { ial cher } \\
\text { er HIFU }\end{array}$ & $\begin{array}{l}\text { logy Group; HI } \\
\text { loembolizatior } \\
\text { treatment; CR, }\end{array}$ & $\begin{array}{l}\text { J, high inter } \\
\text { SOX, oxalip }\end{array}$ & $\begin{array}{l}\text { ity focused } \\
\text { tin and tega }\end{array}$ & $\begin{array}{l}\text { rasound; RF/ } \\
\text { Ir; \# contrast- } \epsilon \\
\text { al }\end{array}$ & radiofrequenc & $\begin{array}{l}\text { ablation; } \\
\text { TT was }\end{array}$ \\
\hline
\end{tabular}

In the BSC group, all patients exhibited tumor progression, only one patient was alive at 15 months, and the remaining 15 patients died from tumor progression. In the PC group, 3 patients achieved CR, 6 patients achieved PR, 3 patients achieved SD and PD occurred in 4 patients. The ORR was $56.3 \%$. One patient was alive at 20 months without any evidence of recurrent disease, and three patients developed either liver recurrence or distant metastasis and were alive. The remaining 12 patients died from tumor progression.

\section{Treatment-related AES}

Acute toxicities that occurred during the HIFU treatment period and for 30 days after completion of the treatment are described in this section. Any grade AEs were observed in four cases. Pain and fatigue were the most common $(n=3)$. Others include fever, increased ALT/AST, and skin edema. Notably, no grade 3 or higher AEs occurred. Table 4 summarized all AEs related to the HIFU treatment. 
Table 4

All any grade adverse events (AEs) related to HIFU treatment after 30 days

\begin{tabular}{|lllll|}
\hline Toxicity & Grade 1 & $\%$ & Grade 2 & $\%$ \\
\hline Any adverse events & 4 & 50.0 & 2 & 25.0 \\
\hline Fatigue & 3 & 37.5 & 0 & - \\
\hline Pain & 3 & 37.5 & 2 & 25.0 \\
\hline Fever & 2 & 25.0 & 0 & - \\
Skin edema & 1 & 12.5 & 0 & - \\
\hline ALT/AST increased & 2 & 25.0 & 1 & 12.5 \\
\hline ALT, alamine aminotransferase; AST, aspartate aminotransferase. \\
\hline No Grade 3 or worse toxicities.
\end{tabular}

In PC, the incidence of any grade AEs was $62.5 \%$ (10/16), and the most common AEs were nausea/vomiting, pain, and fatigue. Other AEs including diarrhea, constipation, peripheral neuropathy, anemia, leukopenia, increased ALT/AST, neutropenia, and thrombocytopenia were also observed. Grade 3-4 AEs accounted for 25\% (4/16), including nausea/vomiting, leukopenia, neutropenia, and thrombocytopenia. No treatment-related deaths were observed.

\section{Discussion}

According to the National Comprehensive Cancer Network guidelines, localized thermal treatment options such as RFA or microwave ablation for liver metastasis of GC are not considered the best supportive treatment option[24]. Systemic chemotherapy is currently the preferred treatment for patients with GCLM. There is no doubt that systemic chemotherapy can improve long-term survival and prolong PFS compared with BSC[25, 26]. TACE and hepatic arterial infusion chemotherapy have also been reported to be used for the treatment of GCLM, but only in small sample retrospective analysis and case reports[27-29]. At present, some scholars have proposed that patients with GCLM can be divided into three categories: potentially resectable tumor (category I), marginally resectable tumor (category II), and unresectable tumor (category III), this determination should be made by an $\operatorname{MDT}[6,30]$. There is also another group of scholars who were inspired by the evidence of substantially increased survival benefits observed in patients who received surgery for colorectal liver metastases and begun to further explore the role of surgery for GCLM[31].

Palliative gastrectomy is currently not recommended for patients with advanced GC (stage IV) unless perforation, bleeding, pyloric obstruction, etc occurs[4, 32]. However, when the metastatic lesions can be removed completely, expanded surgery may improve the patients' prognosis. A retrospective study of 28 patients with GCLM with and without extrahepatic metastasis showed a 5-year survival rate of $32 \%$ after undergoing hepatectomy, with a median OS time of 49 months[9]. Another study involving $25 \mathrm{GC}$ patients with synchronous liver metastasis showed that undergoing hepatectomy resulted in a 5-year recurrence-free survival rate of $11.1 \%$ and a 5 -year OS of $29.4 \%[8]$. It should also be noted that many patients lose the chance of surgery due to either the difficulty of surgical resection or the patients' intolerance to surgery. Fortunately for such patients, RFA can be a viable treatment option for GCLM patients who are unable to undergo hepatectomy. Oki et al. reported that a total of 94 GCLM patients received either surgery or RFA or a combination of both and that the 5-year OS and 5-year recurrence-free survival rate was $42.3 \%$ and $27.7 \%$, respectively[33]. Two observational studies compared the short- and long-term results of RFA with liver resection for 
GCLM patients. The results showed that there was no difference between the 3-year and 5-year survival rates of patients undergoing either RFA and surgery and that RFA had even fewer complications [10,11].

Unfortunately, RFA has several limitations. RFA is not able to treat lesions located near the main biliary tract or gallbladder, major blood vessels, and just beneath the diaphragm[34]. PC is usually used to treat patients with GCLM who are unable to receive either RFA or surgery, but its long-term effects are disappointing. In recent years, HIFU has been increasingly used in the treatment of liver cancer[35,36]. Due to its characteristics, some studies including our previous studies have shown that HIFU can be used as a potential treatment option for patients with liver cancer but are contraindicated for either RFA or surgery $[18,19]$. In a previous study, we reported on 13 patients with colorectal liver metastases who were contraindicated for either resection or RFA and eventually received HIFU treatment. The 2-year PFS was $16.7 \%$, and the median PFS was 9 months. Notably, the 2-year OS was 77.8\%, and the median OS time was 25 months[18]. However, the long-term prognosis of patients with either GCLM or colorectal liver metastases who underwent primary lesion resection combined with hepatectomy is significantly different. The 5-year survival rate of GCLM is about $20 \%[10,37]$, while that of colorectal liver metastases is as high as $50 \%$ after surgery[38, 39]. More importantly, few studies have evaluated the safety and efficacy of HIFU treatment for GCLM patients. Therefore, this is the first study to date that compares the long-term effects of HIFU, PC, and BSC in GCLM patients without extra-hepatic metastasis who were contraindicated for either hepatectomy or RFA. In order to increase the credibility of the study and to reduce the heterogeneity among the treatment groups, propensity matching analysis is used.

In this study, 40 patients with GCLM were included, and the baseline comparisons among the three groups were equivalent. Relevant studies have shown that the potential prognostic factors included but were not limited to age, the number of metastases, and size of metastases[7, 40,41]. Among them, the number of lesions was the most significant prognostic factor. Multiple studies have shown that patients with a single metastasis have a better prognosis than patients with multiple metastases. Fortunately, the three groups in this study were similar in many aspects. In terms of tumor response, due to differences in treatment methods, the patients in the HIFU group were assessed using the mRECIST, while the patients in the PC and BSC groups were evaluated according to RECIST. The results of our study showed that more than half of the patients could achieve CR after HIFU treatment, while less than one-fifth of patients achieved CR in the PC group. In the BSC group, the tumor continued to progress. Similarly, the ORR was higher in the HIFU group than in the PC group. This suggests that HIFU treatment of metastatic lesions is potentially more beneficial compared with PC and BSC. In addition, no rapid tumor progression was observed during the follow-up of the three patients who did not achieve CR.

More importantly, survival analysis showed that patients treated with HIFU had the best OS and PFS, followed by PC, while the BSC group had the worst survival rate. The median survival time in the BSC group was about half a year, which is in line with previous studies[42,43]. The median survival time of the patients who underwent PC was about twice as long as that of the BSC group, up to approximately 1 year. Picado et al. included 3000 patients with GCLM, and the results of this analysis showed that the median survival time of patients receiving PC was about 9.7 months. The median survival time after receiving palliative gastrectomy was 15.3 months, while the median survival time after receiving combined hepatectomy was 24.3 months[40]. Zhang et al.[32] reported that the median survival time for GCLM patients receiving BSC was only 2.8 months, compared with 9.4 months for those receiving systemic chemotherapy. Additionally, patients receiving multi-line chemotherapy had a longer median survival time compared to those receiving single-line chemotherapy (14.2 months versus 6.6 months). A phase II clinical trial showed that capecitabine combined with paclitaxel resulted in a median survival time of 10.1 months for GCLM patients[44]. Therefore, systemic chemotherapy rarely prolongs the median survival time of GCLM patients by more than 2 years.

Interestingly, our research showed that the median survival time of GCLM patients who underwent HIFU treatment was more than 2 years, which was twice that of the PC group, and the median PFS time was more than 1 year, which was 3

Page $13 / 21$ 
times that of the PC group.

HIFU due to its minimal invasiveness and safety characteristics is becoming more widely used in the treatment of liver cancer by inducing precise lesion coagulation necrosis without damaging the surrounding structures. Furthermore, HIFU treatment is reported to have the ability to enhance the patients' tumor immune response [45]. A case of gastric leiomyosarcoma with liver metastases and multiple retroperitoneal lymphatic metastases was reported to be treated with HIFU combined with tetrahydropalmatine and oxaliplatin-based transarterial chemoembolization and the PFS was 4 months after treatment[46]. Park et al. reported in 2009 on 3 GCLM patients who received HIFU treatment, which was generally safe, but the efficacy in that study was indeterminate[47]. It has been reported that a 35-year female patient with metachronous GCLM achieved CR after receiving HIFU combined with chemotherapy[48]. A recent retrospective study showed that HIFU therapy for metastatic liver cancer yielded better long-term results[49]. The one-year survival rate was $48.0 \%$, and the median OS time was 12 months. However, this study had several shortcomings. First, this study included patients with multiple malignant tumors associated with liver metastasis, including colorectal cancer, pancreatic cancer, GC, breast cancer, gallbladder cancer, etc. Second, several patients had extrahepatic metastasis. Therefore, the effect of HIFU on GCLM is not well reflected in this study. Our study preliminarily showed that HIFU treatment has a good long-term effect on patients with GCLM.

In terms of treatment-related AEs, HIFU therapy has been shown to be safe and is usually tolerated well by patients. No patient had grade 3 or above AEs, and other AEs were manageable. After symptomatic treatment, all AEs were alleviated. The AEs associated with HIFU treatment was not significantly worse than the AEs associated with PC.

To the best of our knowledge, this is the first research that specifically compared HIFU treatment with PC and BSC for patients with GCLM by coupled cases. This study evaluated the safety and efficacy of HIFU for patients with GCLM and determined that HIFU treatment can be considered to be a good alternative when surgery or RFA is contraindicated for patients with GCLM. Patients who received HIFU treatment has better long-term outcomes compared to the PC and BSC groups, with no significant increase in AEs.

The limitations of this study are as follows. First, this is a prospective observational study with a small sample size. Although the propensity matching analysis is carried out, the reliability of the conclusion is lacking due to the small sample size. Second, the patients received systemic chemotherapy after HIFU treatment, which may have increased the perceived efficacy of the HIFU treatment. As a result, the therapeutic effect of HIFU may be overestimated. Therefore, this study needs to be further verification by a large sample randomized controlled trial.

\section{Conclusion}

The results of this study showed that HIFU treatment could improve the long-term prognosis of GCLM patients without significantly increasing AEs. Compared with PC and BSC, HIFU is the preferred treatment option when GCLM patients without extra-hepatic metastasis are contraindicated for either surgery or RFA.

\section{Abbreviations}

HIFU, high intensity focused ultrasound; GC, gastric cancer; GCLM, gastric cancer with liver metastasis; BSC, best supportive care; PC, palliative chemotherapy; PFS, progression-free survival; OS, overall survival; RFA, radiofrequency ablation; MDT, multiple disciplinary team; ECOG, Eastern Cooperative Oncology Group; ALT, alanine aminotransferase; AST, aspartate aminotransferase; ULN, upper limit of normal; mRECIST, modified Response Evaluation Criteria in Solid Tumors; TACE, transcatheter arterial chemoembolization; CTCAE, Common Terminology Criteria for Adverse Events; AEs, Adverse events; CR, complete response; PR, partial response; PD, progressive disease; SD, stable disease; ORR, objective response rate; $\mathrm{HR}$, hazard ratios; $\mathrm{Cl}$, confidence intervals; $\mathrm{BMI}$, body mass index. 


\section{Declarations}

\section{Acknowledgements}

None

\section{Conflict of interest}

The authors declare that they have no conflict of interest.

\section{Consent to publication}

Not applicable.

\section{Ethics approval and consent to participate}

All procedures performed in this study were in accordance with the ethical standards of the institutional. The investigation was in accordance with the 1964 Helsinki declaration and its later amendments, the Good Laboratory Practice rules and was approved by the Ethical Committee of the HwaMei Hospital, University of Chinese Academy of Sciences (approval NO.PJ-NBEY-KY-2019-153-01). Informed consent was obtained from all individual participants included in the study. All patients provided a written informed consent before their inclusion in the study.

\section{Availability of data and materials}

The datasets supporting the conclusions of this article are included within the article.

\section{Funding}

This study was funded by the Key Laboratory of Diagnosis and Treatment of Digestive System Tumors of Zhejiang Province (Grant No. 2019E10020), Ningbo Clinical Research Center for Digestive

\section{Authors' contributions}

Each author contributed significantly to concept and development of the present paper. Study conception and design: LHG and QZ. Acquisition of data: LHG, NH, JZH, TY, DMN, XDG and KY. Analysis and interpretation of data: LHG, NH, JZH, XXF, ZZ, PC and JJZ. Writing manuscript: LHG, NH, JZH and QZ. All authors had read and approved the manuscript and ensured that this was the case.

\section{Author information}

Lihu Gu, Ning He and Jiaze Hong equally contributed to this work as co-first authors.

\section{References}

1. Bray F, Ferlay J, Soerjomataram I, Siegel RL, Torre LA, Jemal A: Global cancer statistics 2018: GLOBOCAN estimates of incidence and mortality worldwide for 36 cancers in 185 countries. CA: a cancer journal for clinicians 2018, 68(6):394-424.

2. Bang YJ, Kim YW, Yang HK, Chung HC, Park YK, Lee KH et al: Adjuvant capecitabine and oxaliplatin for gastric cancer after D2 gastrectomy (CLASSIC): a phase 3 open-label, randomised controlled trial. Lancet (London, England) 2012, 379(9813):315-321. 
3. D'Angelica M, Gonen M, Brennan MF, Turnbull AD, Bains $M$, Karpeh MS: Patterns of initial recurrence in completely resected gastric adenocarcinoma. Annals of surgery 2004, 240(5):808-816.

4. Li Z, Fan B, Shan F, Tang L, Bu Z, Wu A et al: Gastrectomy in comprehensive treatment of advanced gastric cancer with synchronous liver metastasis: a prospectively comparative study. World journal of surgical oncology 2015, 13:212.

5. Yu P, Zhang Y: Treatment of Synchronous Liver Metastases from Gastric Cancer: A Single-Center Study. 2020, 12:7905-7911.

6. Luo Z, Rong Z, Huang C: Surgery Strategies for Gastric Cancer With Liver Metastasis. Frontiers in oncology 2019, 9:1353.

7. Chen J, Tang Z, Dong X, Gao S, Fang H, Wu D et al: Radiofrequency ablation for liver metastasis from gastric cancer. European journal of surgical oncology : the journal of the European Society of Surgical Oncology and the British Association of Surgical Oncology 2013, 39(7):701-706.

8. Qiu JL, Deng MG, Li W, Zou RH, Li BK, Zheng Y et al: Hepatic resection for synchronous hepatic metastasis from gastric cancer. European journal of surgical oncology : the journal of the European Society of Surgical Oncology and the British Association of Surgical Oncology 2013, 39(7):694-700.

9. Tatsubayashi T, Tanizawa Y, Miki Y, Tokunaga M, Bando E, Kawamura T et al: Treatment outcomes of hepatectomy for liver metastases of gastric cancer diagnosed using contrastenhanced magnetic resonance imaging. Gastric cancer : official journal of the International Gastric Cancer Association and the Japanese Gastric Cancer Association 2017, 20(2):387-393.

10. Guner A, Son T, Cho I, Kwon IG, An JY, Kim HI et al: Liver-directed treatments for liver metastasis from gastric adenocarcinoma: comparison between liver resection and radiofrequency ablation. 2016, 19(3):951-960.

11. Tang K, Zhang B, Dong L, Wang L, Tang Z: Radiofrequency ablation versus traditional liver resection and chemotherapy for liver metastases from gastric cancer. 2020, 48(7):300060520940509.

12. Kang YK, Boku N, Satoh T, Ryu MH, Chao Y, Kato K et al: Nivolumab in patients with advanced gastric or gastrooesophageal junction cancer refractory to, or intolerant of, at least two previous chemotherapy regimens (ONO-453812, ATTRACTION-2): a randomised, double-blind, placebo-controlled, phase 3 trial. Lancet (London, England) 2017, 390(10111):2461-2471.

13. Fuchs CS, Doi T, Jang RW, Muro K, Satoh T, Machado M et al: Safety and Efficacy of Pembrolizumab Monotherapy in Patients With Previously Treated Advanced Gastric and Gastroesophageal Junction Cancer: Phase 2 Clinical KEYNOTE-059 Trial. JAMA oncology 2018, 4(5):e180013.

14. Hsiao YH, Kuo SJ, Tsai HD, Chou MC, Yeh GP: Clinical Application of High-intensity Focused Ultrasound in Cancer Therapy. Journal of Cancer 2016, 7(3):225-231.

15. Jin C, Zhu H, Wang Z, Wu F, Chen W, Li K et al: High-intensity focused ultrasound combined with transarterial chemoembolization for unresectable hepatocellular carcinoma: long-term follow-up and clinical analysis. European journal of radiology 2011, 80(3):662-669.

16. Diana M, Schiraldi L, Liu YY, Memeo R, Mutter D, Pessaux $P$ et al: High intensity focused ultrasound (HIFU) applied to hepato-bilio-pancreatic and the digestive system-current state of the art and future perspectives. Hepatobiliary surgery and nutrition 2016, 5(4):329-344.

17. Ji Y, Zhang Y, Zhu J, Zhu L, Zhu Y, Hu K et al: Response of patients with locally advanced pancreatic adenocarcinoma to high-intensity focused ultrasound treatment: a single-center, prospective, case series in China. Cancer management and research 2018, 10:4439-4446.

18. Yang T, Ng DM, Du N, He N, Dai X, Chen P et al: HIFU for the treatment of difficult colorectal liver metastases with unsuitable indications for resection and radiofrequency ablation: a phase I clinical trial. 2020. 
19. Zhang L, Zhu H, Jin C, Zhou K, Li K, Su H et al: High-intensity focused ultrasound (HIFU): effective and safe therapy for hepatocellular carcinoma adjacent to major hepatic veins. European radiology 2009, 19(2):437-445.

20. Chen AP, Setser A, Anadkat MJ, Cotliar J, Olsen EA, Garden BC et al: Grading dermatologic adverse events of cancer treatments: the Common Terminology Criteria for Adverse Events Version 4.0. Journal of the American Academy of Dermatology 2012, 67(5):1025-1039.

21. Lencioni R, Llovet JM: Modified RECIST (mRECIST) assessment for hepatocellular carcinoma. Seminars in liver disease 2010, 30(1):52-60.

22. Eisenhauer EA, Therasse P, Bogaerts J, Schwartz LH, Sargent D, Ford R et al: New response evaluation criteria in solid tumours: revised RECIST guideline (version 1.1). European journal of cancer (Oxford, England : 1990) 2009, 45(2):228-247.

23. Gu L, Chen P, Su H, Li X, Zhu H, Wang X et al: Clinical Significance of Tumor Deposits in Gastric Cancer: a Retrospective and Propensity Score-Matched Study at Two Institutions. 2020, 24(11):2482-2490.

24. Ajani JA, D'Amico TA, Almhanna K, Bentrem DJ, Chao J, Das P et al: Gastric Cancer, Version 3.2016, NCCN Clinical Practice Guidelines in Oncology. Journal of the National Comprehensive Cancer Network : JNCCN2016, 14(10):1286-1312.

25. Mitachi Y, Sakata Y, Ohtsu A, Hyodo I, Katsu K, Sairenji M et al: Docetaxel and cisplatin in patients with advanced or recurrent gastric cancer: a multicenter phase I/II study. Gastric cancer : official journal of the International Gastric Cancer Association and the Japanese Gastric Cancer Association 2002, 5(3):160-167.

26. Kakeji Y, Morita M, Maehara Y: Strategies for treating liver metastasis from gastric cancer. Surgery today 2010, 40(4):287-294.

27. Toyokawa T, Ohira M, Sakurai K, Amano R, Kubo N, Tanaka H et al: Long-term survival with complete remission after hepatic arterial infusion chemotherapy for liver metastasis from gastric cancer: a case report. World journal of surgical oncology 2015, 13:268.

28. Moriya A, Hyodo I, Nishina T, Imaoka H, Imagawa A, Doi T et al: Extensive liver metastasis of gastric cancer effectively treated by hepatic arterial infusion of 5-fluorouracil/cisplatin. Gastric cancer : official journal of the International Gastric Cancer Association and the Japanese Gastric Cancer Association 2000, 3(2):110-115.

29. Vogl TJ, Gruber-Rouh T, Eichler K, Nour-Eldin NE, Trojan J, Zangos S et al: Repetitive transarterial chemoembolization (TACE) of liver metastases from gastric cancer: local control and survival results. European journal of radiology 2013, 82(2):258-263.

30. Yoshida K, Yamaguchi K, Okumura N, Tanahashi T, Kodera Y: Is conversion therapy possible in stage IV gastric cancer: the proposal of new biological categories of classification. Gastric cancer : official journal of the International Gastric Cancer Association and the Japanese Gastric Cancer Association 2016, 19(2):329-338.

31. Hur H, Ko YT, Min BS, Kim KS, Choi JS, Sohn SK et al: Comparative study of resection and radiofrequency ablation in the treatment of solitary colorectal liver metastases. American journal of surgery 2009, 197(6):728-736.

32. Zhang W, Yu Y, Fang Y, Wang Y, Cui Y, Shen K et al: Systemic chemotherapy as a main strategy for liver metastases from gastric cancer. Clinical \& translational oncology : official publication of the Federation of Spanish Oncology Societies and of the National Cancer Institute of Mexico 2015, 17(11):888-894.

33. Oki E, Tokunaga S, Emi Y, Kusumoto T, Yamamoto M, Fukuzawa K et al: Surgical treatment of liver metastasis of gastric cancer: a retrospective multicenter cohort study (KSCC1302). Gastric cancer : official journal of the International Gastric Cancer Association and the Japanese Gastric Cancer Association 2016, 19(3):968-976.

34. Aliyev S, Agcaoglu O, Aksoy E, Taskin HE, Vogt D, Fung J et al: Efficacy of laparoscopic radiofrequency ablation for the treatment of patients with small solitary colorectal liver metastasis. Surgery 2013, 154(3):556-562. 
35. Ng KK, Poon RT, Chan SC, Chok KS, Cheung TT, Tung H et al: High-intensity focused ultrasound for hepatocellular carcinoma: a single-center experience. Annals of surgery 2011, 253(5):981-987.

36. Cheung TT, Poon RT, Jenkins CR, Chu FS, Chok KS, Chan AC et al: Survival analysis of high-intensity focused ultrasound therapy vs. transarterial chemoembolization for unresectable hepatocellular carcinomas. Liver international : official journal of the International Association for the Study of the Liver 2014, 34(6):e136-143.

37. Shirasu H, Tsushima T, Kawahira M, Kawai S, Kawakami T, Kito Y et al: Role of hepatectomy in gastric cancer with multiple liver-limited metastases. Gastric cancer : official journal of the International Gastric Cancer Association and the Japanese Gastric Cancer Association 2018, 21(2):338-344.

38. Pawlik TM, Scoggins CR, Zorzi D, Abdalla EK, Andres A, Eng C et al: Effect of surgical margin status on survival and site of recurrence after hepatic resection for colorectal metastases. Annals of surgery 2005, 241(5):715-722, discussion 722-714.

39. Imai K, Allard MA, Castro Benitez C, Vibert E, Sa Cunha A, Cherqui D et al: Long-term outcomes of radiofrequency ablation combined with hepatectomy compared with hepatectomy alone for colorectal liver metastases. The British journal of surgery 2017, 104(5):570-579.

40. Picado O, Dygert L, Macedo FI, Franceschi D, Sleeman D, Livingstone AS et al: The Role of Surgical Resection for Stage IV Gastric Cancer With Synchronous Hepatic Metastasis. The Journal of surgical research 2018, 232:422-429.

41. Li J, Xi H, Cui J, Zhang K, Gao Y, Liang W et al: Minimally invasive surgery as a treatment option for gastric cancer with liver metastasis: a comparison with open surgery. Surgical endoscopy 2018, 32(3):1422-1433.

42. Yagi Y, Seshimo A, Kameoka S: Prognostic factors in stage IV gastric cancer: univariate and multivariate analyses. Gastric cancer : official journal of the International Gastric Cancer Association and the Japanese Gastric Cancer Association 2000, 3(2):71-80.

43. Wu P, Wang P, Ma B, Yin S, Tan Y, Hou W et al: Palliative gastrectomy plus chemotherapy versus chemotherapy alone for incurable advanced gastric cancer: a meta-analysis. Cancer management and research 2018, 10:4759-4771.

44. Wu AW, Yuan P, Li ZY, Tang L, Bu ZD, Ren $\mathrm{H}$ et al: Capecitabine plus paclitaxel induction treatment in gastric cancer patients with liver metastasis: a prospective, uncontrolled, open-label Phase II clinical study. Future oncology (London, England) 2016, 12(18):2107-2116.

45. Maloney E, Hwang JH: Emerging HIFU applications in cancer therapy. International journal of hyperthermia : the official journal of European Society for Hyperthermic Oncology, North American Hyperthermia Group 2015, 31(3):302-309.

46. Cheng CS, Chen L, Xie J, Chen Z: Multimodality palliative treatment with transarterial chemoembolization and highintensity focused ultrasound for gastric leiomyosarcoma multiple liver metastasis pain: A case report. Medicine 2019, 98(39):e17328.

47. Park MY, Jung SE, Cho SH, Piao XH, Hahn ST, Han JY et al: Preliminary experience using high intensity focused ultrasound for treating liver metastasis from colon and stomach cancer. International journal of hyperthermia : the official journal of European Society for Hyperthermic Oncology, North American Hyperthermia Group 2009, 25(3):180-188.

48. Sung HY, Cho SH, Kim JI, Cheung DY, Han JY, Kim JK et al: High intensity focused ultrasound therapy resulted in a complete response in a patient with advanced gastric cancer with liver metastases: a case report. European journal of gastroenterology \& hepatology 2008, 20(7):707-709.

49. Ji Y, Zhu J, Zhu L, Zhu Y, Zhao H: High-Intensity Focused Ultrasound Ablation for Unresectable Primary and Metastatic Liver Cancer: Real-World Research in a Chinese Tertiary Center With 275 Cases. Frontiers in oncology 2020, 10:519164. 


\section{Figures}

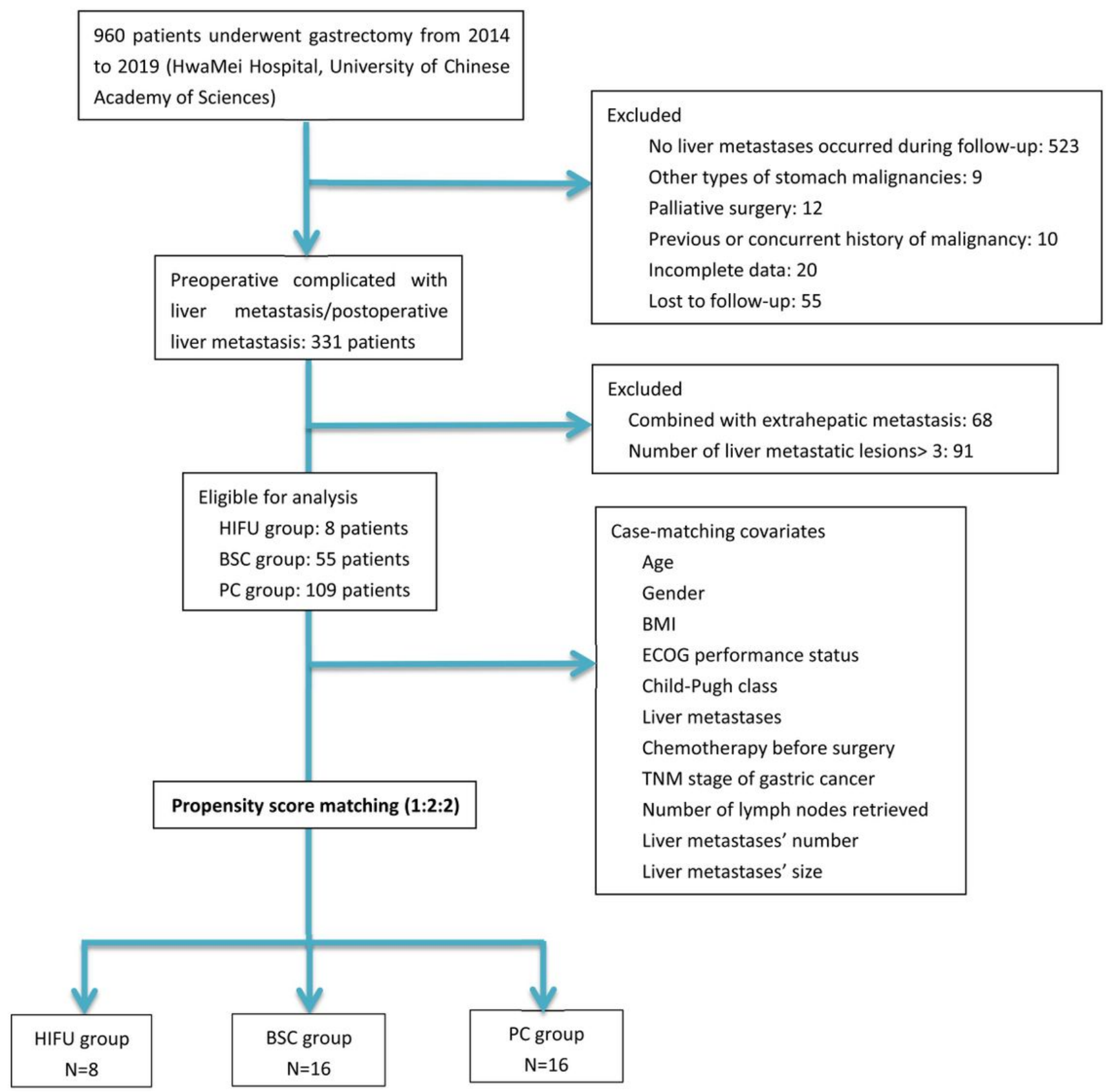

Figure 1

Flowchart of the study population. 


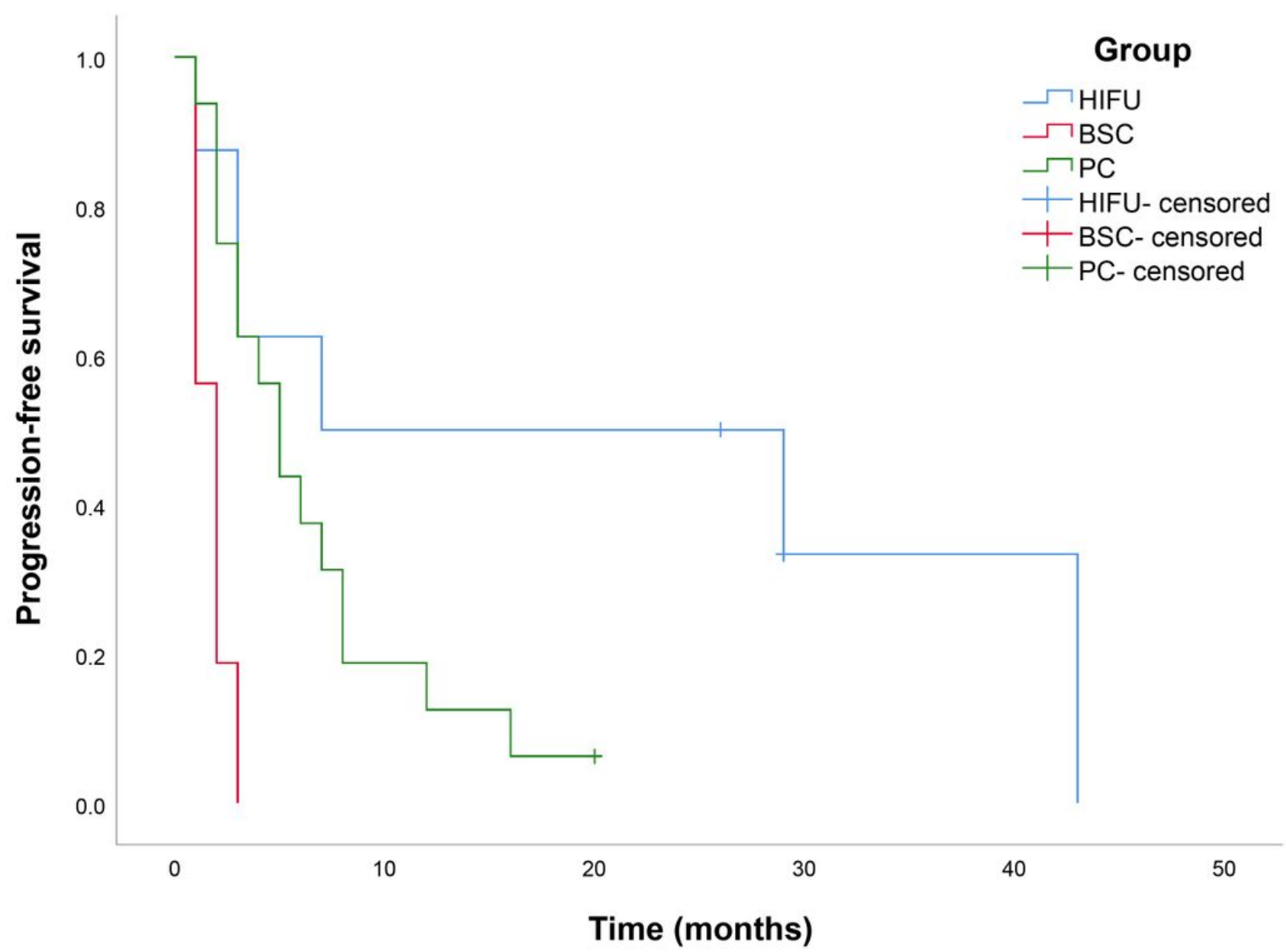

No. of patient at risk

$\begin{array}{rcccccc}\text { HIFU } & 8 & 4 & 4 & 1 & 1 & 0 \\ \text { BSC } & 16 & 0 & 0 & 0 & 0 & 0 \\ \text { PC } & 16 & 3 & 1 & 0 & 0 & 0\end{array}$

Figure 2

Comparison of survival curves for progression-free survival (PFS) among HIFU group, BSC group, and PC group. $(p<0.001)$ 


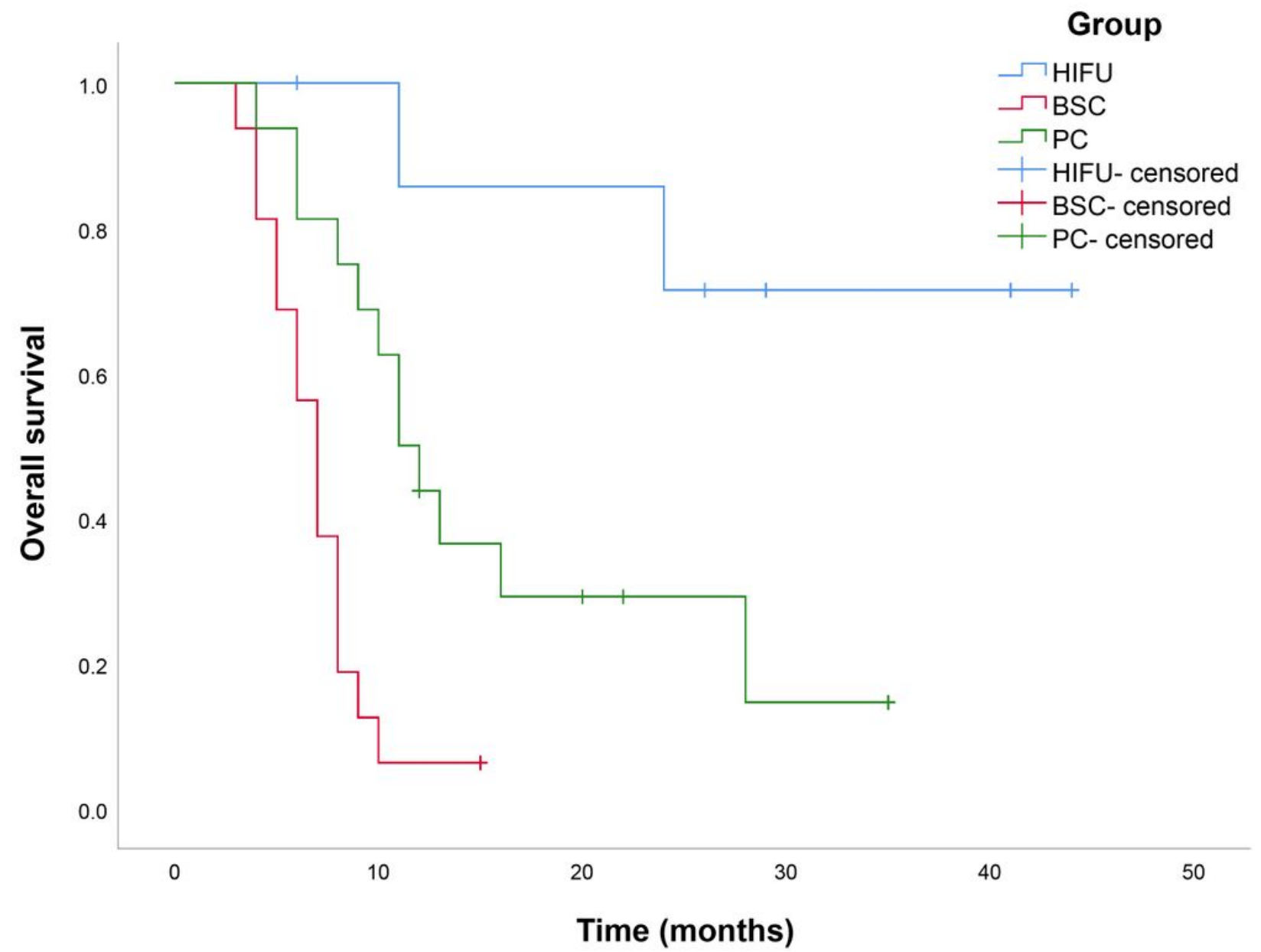

No. of patient at risk

$\begin{array}{rrrrrrr}\text { HIFU } & 8 & 7 & 6 & 3 & 3 & 0 \\ \text { BSC } & 16 & 2 & 0 & 0 & 0 & 0 \\ \text { PC } & 16 & 11 & 4 & 1 & 0 & 0\end{array}$

Figure 3

Comparison of survival curves for overall survival (OS) among HIFU group, BSC group, and PC group. $(p<0.001)$

\section{Supplementary Files}

This is a list of supplementary files associated with this preprint. Click to download.

- SupplementaryTable1.docx 